\title{
Stochastic Integral Equation Solver for Efficient Variation-Aware Interconnect Extraction
}

\author{
Tarek El-Moselhy \\ Computational Prototyping Group \\ Research Laboratory in Electronics \\ Massachusetts Institute of Technology \\ Cambridge, MA 02139 \\ tmoselhy@mit.edu
}

\author{
Luca Daniel \\ Computational Prototyping Group \\ Research Laboratory in Electronics \\ Massachusetts Institute of Technology \\ Cambridge, MA 02139 \\ luca@mit.edu
}

\begin{abstract}
In this paper we present an efficient algorithm for extracting the complete statistical distribution of the input impedance of interconnect structures in the presence of a large number of random geometrical variations. The main contribution in this paper is the development of a new algorithm, which combines both Neumann expansion and Hermite expansion, to accurately and efficiently solve stochastic linear system of equations. The second contribution is a new theorem to efficiently obtain the coefficients of the Hermite expansion while computing only low order integrals. We establish the accuracy of the proposed algorithm by solving stochastic linear systems resulting from the discretization of the stochastic volume integral equation and comparing our results to those obtained from other techniques available in the literature, such as Monte Carlo and stochastic finite element analysis. We further prove the computational efficiency of our algorithm by solving large problems that are not solvable using the current state of the art.
\end{abstract}

Categories and Subject Descriptors: G.1.9 [Integral Equations]; J.6 [Computer-Aided Engineering]: Computer-aided design (CAD) General Terms: Algorithms, Theory, Design

Keywords: Stochastic field solvers, Polynomial chaos expansion, Neumann expansion, surface roughness, variation-aware extraction

\section{INTRODUCTION}

On-chip and off-chip fabrication processes may typically generate interconnect structures of irregular geometries. Such irregularities are not deterministic, and are produced by several different manufacturing steps such as etching, chemical mechanical polishing (CMP), electro-deposition, and photolithography. The effect of the geometrical uncertainties on the electrical characteristics of the interconnect structures has been widely ignored during the past decade since it was not significant. However, as a result of technology scaling, such manufacturing uncertainties are now beginning to play a major roles in determining the electrical characteristics of the interconnect structures. During the last few years several attempts have been proposed to develop efficient stochastic solvers $[1,2$,

Permission to make digital or hard copies of all or part of this work for personal or classroom use is granted without fee provided that copies are not made or distributed for profit or commercial advantage and that copies bear this notice and the full citation on the first page. To copy otherwise, or republish, to post on servers or to redistribute to lists, requires prior specific permission and/or a fee.

DAC 2008, June 8-13, 2008, Anaheim, California, USA.

Copyright 2008 ACM 978-1-60558-115-6/08/0006...5.00.
3]. In [1] an algorithm has been proposed to obtain an approximation of the average and the standard deviation of the capacitance of off-chip interconnect structures with surface roughness. However, the technique is computationally very expensive $O\left(N^{4}\right)$, and required the development of complex sparsification techniques [4]. Despite that effort such technique can only compute the average and the standard deviation of the electrostatic capacitance, and cannot be generalized to compute the complete distribution of the capacitance. In [2] a more general algorithm for extracting the complete distribution of the electrostatic capacitance of on-chip interconnects is proposed. However, the algorithm is also computationally very expensive and cannot handle large size problems. In [3] another computationally expensive $O\left(N^{4}\right)$ algorithm based on [1] is presented to extract the input impedance of off-chip interconnects with surface roughness under magneto-quasistatic assumption. Similar to [1] the algorithm cannot be generalized to extract complete distributions.

The inherent difficulties facing the above algorithms is better understood if we abstract the problem of extracting the input impedance of interconnect structures with a large number of correlated random variations to one of solving a large linear system of the form $A(\eta) x(\eta)=b(\eta)$, where $\eta$ is a large vector of random variables. Several techniques have been proposed before to address this problem, such as Monte-Carlo or Monte-Carlo-like methods [5, 2], Neumann expansion [1, 3], and stochastic finite element simulation (SFE) [6]. However, none of these techniques is practical for large size problems, typically encountered when the ratio of the physical geometrical domain bounding the irregularities of the interconnect structure is large compared to the correlation length of the random processes. In such case the number of independent random variables required to simulate the random process (length of $\eta)$ becomes very large and all of the above techniques become inefficient. Nevertheless, the challenges facing each technique are different. For Monte Carlo based methods [2] the convergence becomes very slow and therefore accurate results require a very large number of system solves, making the problem computationally not feasible for any practical interconnect structure. For Neumann expansion based algorithms $[1,3]$ calculating the statistics of the high order terms becomes computationally very expensive. In addition, calculating the complete distribution is not possible. For SFE [6] the linear system size becomes very large and therefore unsolvable. Another computational difficulty, which in general is associated with algorithms that rely on expanding the random functions in terms of orthogonal basis functions, such as [5, 2, 6], is the need to compute integrals of very large dimension. Such requirement is limiting the usability of such algorithms in electrical extraction problems of large size.

In this paper we present a methodology that combines the strengths of the above approaches and avoids their weaknesses to efficiently 
extract the complete statistical distribution of the input impedance of interconnect structures with geometrical variations. For the sake of clarity we will restrict our discussion to volume integral equations with surface roughness, which is the most difficult large problem, since the interconnect surface typically spans several hundred or thousand correlation lengths. However, our algorithm can also be applied to simulating on-chip geometrical variations.

In the next section we will summarize the mathematical description of the problem. In section 3 we develop a new theorem to efficiently compute the coefficients of the expansion of a random function in terms of a set of orthogonal polynomials without computing any high dimensional integrals. In section 4 we present a new simulation method in which the new expansion technique is integrated with the Neumann expansion to compute the complete stochastic characteristics of the unknown vector. Finally, in section 5 we establish the accuracy and computational efficiency of such stochastic simulation methodology.

\section{BACKGROUND}

\subsection{Formulation}

We use the standard mixed potential integral equation to describe the relation between the volumetric current density $\bar{J}(r)$ inside the conductor, the surface charge distribution $\rho(r)$, and the electric potential $\phi(r)[7]$ :

$$
\begin{aligned}
\frac{\bar{J}(r)}{\sigma_{c}}+j \omega \frac{\mu}{4 \pi} \int_{V} G\left(r, r^{\prime}\right) \bar{J}\left(r^{\prime}\right) d r^{\prime} & =-\nabla \phi(r) \\
\frac{1}{4 \pi \varepsilon} \int_{S} G\left(r, r^{\prime}\right) \rho\left(r^{\prime}\right) d r^{\prime} & =\phi(r) \\
\nabla \cdot \bar{J}(r) & =0 \\
\hat{n} \cdot \bar{J}(r) & =j \omega \rho(r),
\end{aligned}
$$

where $G\left(r, r^{\prime}\right)$ is the free space Green's function, $V, S$ are the conductors volume and rough surface area, respectively, $\sigma_{c}$ is the conductor conductivity, $\varepsilon$ is the complex dielectric constant including dielectric losses, $\mu$ is the magnetic permeability, $\omega$ is the angular frequency in radians.

\subsection{Rough Surface Model}

The volume of the conductors $V$ is bounded by rough surfaces $S$ that we assume described by a stochastic Gaussian process and a Gaussian correlation function:

$$
P(H(R))=\frac{\exp \left(-0.5 H^{T} \Sigma(R)^{-1} H\right)}{(2 \pi)^{0.5 n} \sqrt{|\Sigma(R)|}}, \quad \Sigma(R)_{i j}=\sigma^{2} \exp \left(-\frac{\left\|r_{i}-r_{j}\right\|^{2}}{L_{c}^{2}}\right)
$$

where $H(R) \in \mathbb{R}^{n \times 1}$ is a vector of surface heights at locations given by vector $R \in \mathbb{R}^{n \times 1}$, i.e. $H\left(r_{1}\right)$ is the surface height at location $r_{1}$. $\Sigma \in \mathbb{R}^{n \times n}$ is the correlation matrix.

\subsection{Linear System Assembly}

A standard procedure for solving (1) involves discretizing the current density $\bar{J}(r)$ and the charge density $\rho(r)$ using some basis functions. Then a Galerkin technique is used to obtain the resistance, partial inductance, and potential matrices $R, L$ and $P$ [7]. The current conservation constraint in (3) can be imposed using mesh analysis to obtain a linear system:

$$
M Z M^{T} I_{m}=\Delta \Phi_{m},
$$

where $M$ is the mesh incidence matrix, $\Delta \Phi_{m}$ is the known RHS vector of branch voltages, $I_{m}$ is the unknown mesh current, and $Z$ is the known branch impedance matrix:

$$
Z=\left[\begin{array}{cc}
R+j \omega L & 0 \\
0 & \frac{P}{j \omega}
\end{array}\right],
$$

The matrix elements of the matrix $Z$ are given by:

$$
R_{i i}=\int_{V_{i}} \frac{\rho}{A_{i} A_{i}} d^{3} r, \quad L_{i j}=\int_{V_{j} V_{i}} \frac{G\left(r, r^{\prime}\right)}{A_{i} A_{j}} d^{3} r^{\prime} d^{3} r, \quad P_{i j}=\int_{S_{j}} \int_{S_{i}} \frac{G\left(r, r^{\prime}\right)}{S_{i} S_{j}} d^{2} r^{\prime} d^{2} r,
$$

where $V_{i}, A_{i}$ are the volume and cross sectional area of filament $i$, respectively, and $S_{i}$ is the panel surface area of panel $i$.

The most important observation at this point is that the integration domain is described by the rough surfaces and that the integrals are therefore stochastic functions of the random process. To emphasize this dependence we will re-write (5) as $M Z(H) M^{T} I_{m}(H)=$ $\Delta \Phi_{m}$, where $H$ is the random process describing the rough surface. Note that we have dropped the $R$ dependence to simplify the notation. Consequently, we have formed the stochastic linear system equivalent to that of the abstract problem $A(\eta) x(\eta)=b$. The objective is to obtain the complete distribution of the mesh current vector $I_{m}(H)$.

\subsection{Solving the Stochastic Linear System (5)}

In this section we summarize the three standard techniques proposed in literature for solving the stochastic linear system (5)

\subsubsection{Monte Carlo}

The system (5) is solved $N_{M C}$ times for different realization of the random process $H$ to obtain the set of solutions $\left\{I_{m}\left(H_{1}\right), I_{m}\left(H_{2}\right)\right.$, $\left.\cdots, I_{m}\left(H_{N_{M C}}\right)\right\}$. The complete statistical distribution of $I_{m}(H)$ is then constructed from the obtained solution set. However, the convergence is very slow $O\left(\frac{1}{\sqrt{N_{M C}}}\right)$ and therefore requires a very large number of system solves.

\subsubsection{Neumann Expansion}

The known stochastic system matrix $Z_{m}(H)=M Z(H) M^{T}$ is first written as $Z_{m}(H)=Z_{m 0}+\Delta Z_{m}(H)$, a sum of a deterministic expectation matrix $Z_{m 0}=E\left(Z_{m}(H)\right)$ and a stochastic matrix $\Delta Z_{m}(H)$. The next step is to substitute the two terms expression for $Z_{m}(H)$ in the linear system (5) and expand the inverse using the Neumann expansion to obtain:

$$
\begin{aligned}
I_{m}(H) & =I_{m 0}-Z_{m 0}^{-1} \Delta Z_{m}(H) I_{m 0} \\
& +Z_{m 0}^{-1} \Delta Z_{m}(H) Z_{m 0}^{-1} \Delta Z_{m}(H) I_{m 0}+\ldots
\end{aligned}
$$

where $I_{m 0}=Z_{m 0}^{-1} \Delta \Phi_{m}$. All of the statistics of the unknown current vector $I_{m}(H)$ can then be obtained from (6). The fundamental difficulty in this algorithm is that calculating the statistics (or even just the average) of high order terms is computationally very expensive $[1,3]$.

\subsubsection{Stochastic Finite Element Simulation}

The stochastic finite element simulation [6] can be used to obtain the complete distribution of the mesh current in (5). The process can be summarized in three steps. First, a Karhunen Loeve expansion is used to expand the random process as a summation of independent random variables [8],

$$
H(r)=\sum_{m=0}^{M} \alpha_{m} \phi_{m}(r) \eta_{m},
$$

where in our particular problem $H(r)$ is the rough surface height at the location $r$, and $\eta_{m}$ is the set of independent Gaussian random variables. Symbols $\alpha_{m}, \phi(r)$ are the square roots of the eigenvalues, and the eigenfunctions of the correlation kernel $C\left(r, r^{\prime}\right)=$ $\sigma^{2} \exp \left(-\frac{\left\|r-r^{\prime}\right\|^{2}}{L_{c}^{2}}\right)$, respectively. The elements of the matrix $Z(H)$ in (5) are then expressed as a function of the expanded random pro- 
cess:

$$
Z_{i j}(H)=Z_{i j}\left(\sum_{m=0}^{M} \alpha_{m} \phi_{m}(r) \eta_{m}\right)
$$

As a second step, a polynomial chaos expansion is used to expand (7) as a series of orthogonal basis polynomials $\Psi_{k}(\eta)$. A complete Askey scheme has been developed [9] to choose the set of orthogonal polynomials in such a way to be compatible with the set of independent random variables $\eta_{m}$. For the particular case of Gaussian random variables the set of Hermite polynomials is the standard choice. The multivariate polynomial expansion step is computationally very expensive and involves integrals of large dimensions.

$$
\begin{aligned}
Z_{i j}(H) & =\sum_{k=0}^{K} Z_{i j}^{k} \Psi_{k}(\eta) \\
Z_{i j}^{k} & =<Z_{i j}(H), \Psi_{k}(\eta)> \\
& =\int_{\eta} Z_{i j}\left(\sum_{m=0}^{M} \alpha_{m} \phi_{m}(r) \eta_{m}\right) \Psi_{k}(\eta) \frac{\exp \left(-\frac{\eta^{T} \eta}{2}\right)}{(2 \pi)^{\frac{M}{2}}} d^{M} \eta .
\end{aligned}
$$

Despite the fact that the exponential function in (8) is separable, the fact that $Z_{i j}(H)$ in (7) is dependent on all the random variables used to expand the random process results in an integral (8) of dimension $M$ independent of $k$. Such large dimensional integrals are computationally very challenging to evaluate. Several techniques have been proposed to avoid this inherent complexity such as Monte Carlo integration (not to be confused with Monte Carlo simulation) and sparse grid integrations [10]. Nevertheless, the problem of calculating a large number of such integrals in a computationally efficient framework remains one of the bottlenecks of stochastic finite element simulation. In section 3 we propose a new theorem to overcome this inherent difficulty.

In the third and final step the expansion of the individual matrix element are assembled together to yield an expansion for the mesh impedance matrix $M Z(H) M^{T}=\sum_{i=0}^{K} Z_{i} \Psi_{i}(\eta)$ and the unknown mesh current is written as an expansion of the same orthogonal polynomials $I_{m}(H)=\sum_{j=0}^{K} I_{j} \Psi_{j}(\eta)$. Both are then substituted in (5) and a Galerkin testing, i.e. projection on the space of the same set of orthogonal polynomials, is applied to obtain a linear system of equations.

$$
\begin{aligned}
\sum_{i=0}^{K} \sum_{j=0}^{K}\left\langle\Psi_{i}(\eta) \Psi_{j}(\eta), \Psi_{\ell}(\eta)\right\rangle Z_{i} I_{j} & =\left\langle\Delta \Phi_{m}, \Psi_{\ell}(\eta)\right\rangle \\
\forall \ell & \in\{1, \cdots, K\}
\end{aligned}
$$

Equation (9) is equivalent to a linear system of the form $A x=b$, where

$$
\begin{gathered}
A=\sum_{i=0}^{K}\left(\left(\begin{array}{cccc}
\gamma_{i 00} & \gamma_{i 10} & \cdots & \gamma_{i K 0} \\
\gamma_{i 01} & \gamma_{i 11} & \cdots & \gamma_{i K 1} \\
& & \ddots & \\
\gamma_{i 0 K} & \gamma_{i 1 K} & \cdots & \gamma_{i K K}
\end{array}\right) \otimes Z_{i}\right) \\
x=\left(\begin{array}{llll}
I_{0} & I_{1} & \cdots & I_{K}
\end{array}\right)^{T}, \quad b=\left(\begin{array}{lllll}
\Delta \Phi_{m} & 0 & \cdots & 0
\end{array}\right)^{T}
\end{gathered}
$$

where $\gamma_{i j \ell}=\left\langle\Psi_{i}(\eta) \Psi_{j}(\eta), \Psi_{\ell}(\eta)\right\rangle$. The size of this linear system is $O(N K)$, i.e. the original system size $N$ times $K$ the total number of multivariate orthogonal polynomials used to expand the random function. $K$ is actually a function of both $P$, the order of the orthogonal polynomial expansion, and $M$, the number of dominant eigenfunctions obtained from the Karhunen Loeve expansion [11]:

$$
K=1+\sum_{i=1}^{P}\left(\begin{array}{c}
i+M-1 \\
i
\end{array}\right) .
$$

There are two main computational challenges involved with the standard stochastic finite element simulation technique when the number of independent random variables $M$, required to represent the random process, is larger than a few variables:

1. The impractical large dimension of the integral in (8).

2. The impractical large size of the linear system in (9).

In section 3 we solve the first problem. In section 4 we present a new methodology that overcomes the second challenge.

\section{MODIFIED HERMITE EXPANSION}

To overcome the main computational difficulty associated with applying polynomial chaos expansion, (namely the need to calculate integrals of very high dimensionality), we present a new theorem summarizing a novel technique to implement the polynomial chaos expansion.

THEOREM 1. Let $H \in \mathbb{R}^{D \times 1}$ be a Gaussian correlated random vector, $\eta \in \mathbb{R}^{M \times 1}$ a vector of $M$ Gaussian independent random variables, and $\Psi(\eta)$ the set of orthogonal Hermite polynomials. Assume that the correlation matrix between $H$ and $\eta$ is known, then the modified Hermite expansion of the function $f(H): \mathbb{R}^{D \times 1} \mapsto \mathbb{R}$ in terms of $\Psi(\eta)$ is:

$$
f(H)=\sum_{i=0}^{K}<f(H), \Psi_{i}\left(\zeta_{i}\right)>\Psi_{i}\left(\zeta_{i}\right)
$$

where

$$
\begin{gathered}
<f(H), \Psi_{i}\left(\zeta_{i}\right)>=\iint f(H) \Psi_{i}\left(\zeta_{i}\right) \frac{\exp \left(-0.5 V^{T} C_{V}^{-1} V\right)}{(2 \pi)^{\frac{D+i}{2}} \sqrt{\left|C_{V}\right|}} d H d \zeta_{i} \\
V=\left(\begin{array}{c}
H \\
\zeta_{i}
\end{array}\right), \quad C_{V}=\left(\begin{array}{cc}
C_{H} & <H, \zeta_{i}^{T}> \\
<\zeta_{i}, H^{T}> & C_{\zeta_{i}}
\end{array}\right)
\end{gathered}
$$

where $\zeta_{i} \in \mathbb{R}^{i \times 1}$ is an at most $i$ element subvector of $\eta$ on which the $i^{\text {th }}$ order Hermite polynomial $\Psi_{i}\left(\zeta_{i}\right)=\Psi_{i}(\eta)$ depends, $C_{H}$ is the correlation matrix associated with the random vector $H, C_{\zeta_{i}}$ is the diagonal correlation matrix associated with $\zeta_{i}$ and $\left|C_{V}\right|$ is the determinant of the correlation matrix $C_{V}$.

PROOF. The main step of the proof is to demonstrate that using the weighting function $P(H, \eta)=\frac{\exp \left(-0.5 V^{T} C_{V}^{-1} V\right)}{(2 \pi)^{\frac{D+M}{2}} \sqrt{\left|C_{V}\right|}}$ the orthogonality of the Hermite polynomials is still preserved. In this step we rely on the interpretation of the weighting function as a probability density function and on the fact that the integral of the multivariate probability density function with respect to any subset of its random variables is the probability density function for the remaining subset of random variables. The second step is to show that if $f(H)=\sum_{i} f_{i} \Psi_{i}\left(\zeta_{i}\right)$ then $f_{i}=<f(H), \Psi_{i}\left(\zeta_{i}\right)>$, i.e. satisfies (11). This is done by multiplying the expansion for $f(H)$ by $\Psi_{i}\left(\zeta_{i}\right)$ and making use of the orthogonality of the Hermite polynomials to compute the required inner product. We also make use of the probability density function interpretation of the weighting function and the fact that by definition $\Psi_{i}(\eta)=\Psi_{i}\left(\zeta_{i}\right)$. An alternative proof is to show that (11) is equivalent to (8), which can be established via a variable transformation. The details of both proofs are omitted due to the lack of space. 
An alternative proof is to show that (11) is equivalent to (8), which can be established via a variable transformation. The details of the alternative proofs are omitted due to the lack of space.

COROLlaRY 1. The maximum dimension of the integrals required to obtain the modified Hermite expansion is $D+P$, where $D$ is the length of vector $H$ and $P$ is the order of the Hermite polynomial. In other words, the dimension of the integral is independent of the number of orthogonal random variables $M$ used for expanding the random process.

PROOF. The proof follows directly from the application of Theorem 1, however, it remains to be shown that the correlation matrix $\left\langle H, \zeta_{p}^{T}\right\rangle$ is known. The calculation of this correlation matrix depends on the particular technique used to generate the set of basis random variables $\eta$, however, for any KL-based expansion we can write component $i$ of vector $H$ as $H_{i}=\sum_{m=0}^{M} \alpha_{m} \phi_{m}\left(r_{i}\right) \eta_{m}$. Consequently, the matrix element $\left\langle H, \zeta_{p}^{T}\right\rangle(i, j)$ :

$$
\begin{aligned}
\left\langle H, \zeta_{p}^{T}\right\rangle(i, j) & =\sum_{m=0}^{M} \alpha_{m} \phi_{m}\left(r_{i}\right)\left\langle\eta_{m}, \zeta_{p}(j)\right\rangle \\
& =\alpha_{\zeta_{p}(j)} \phi_{\zeta_{p}(j)}\left(r_{i}\right) \sigma_{\zeta_{p}(j)},
\end{aligned}
$$

where $\zeta_{p}(j)$ is the $j^{t h}$ element of the random vector $\zeta_{p}$ and $\alpha_{\zeta_{p}(j)}$, $\phi_{\zeta_{p}(j)}(r)$ and $\sigma_{\zeta_{p}(j)}$ are the eigenvalue, eigenfunction and variance associated with the random variable $\zeta_{p}(j)$, respectively. The existence of these quantities is a direct consequence of the fact that $\zeta_{p}$ is a subvector of $\eta$.

The main computational advantage of using Theorem 1 to compute the coefficients of the Hermite chaos expansion stems from the fact that $M$, the total length of the vector $\eta$, is typically 100 , whereas $D$, the length of the height vector, is 2 (since the height vector contains only two components, one for the source filament and one for the observation filament), and $P$, the order of the expansion, is typically 2 (since a second order expansion is sufficient for most applications). Consequently, using Theorem 1 the dimension of the integral for a second order polynomial expansion is reduced from 100 to 4 . In terms of required computation time Theorem 1 accelerates the evaluation of the coefficients as compared to the standard tensor rule of a Q-point quadrature scheme by a factor of $Q^{96}$ (for $\mathrm{Q}=8$ this corresponds to $5 \times 10^{86}$ ), and as compared to Monte Carlo integration or sparse grid integrations by a factor of $\frac{(2 M)^{Q}}{Q ! Q^{4}}$ (for $\mathrm{M}=100, \mathrm{Q}=8$ this corresponds to $1.5 \times 10^{10}$ ).

\section{COMBINED NEUMANN - HERMITE CHAOS EXPANSION}

Despite resolving the computational difficulties associated with finding the coefficients of the expansion using Theorem 1, the stochastic finite element simulation still requires solving a linear system which is $K$ times larger than the original system, where the number of orthogonal polynomials $K$ can be as large as 5000 for a typical large application. The complexity of solving the system will be $K^{2}$ more expensive for an iterative solver and $K^{3}$ more expensive for a direct solver. Such an increase in complexity will result in an inefficient algorithm, possibly even worse that Monte Carlo simulation. On the other hand, we know that the complexity with Neumann expansion is not associated with the system size but rather with calculating the statistics of the high order terms of the expansion. We therefore propose to use Neumann expansion (6) to remove the complexity of solving a large linear system and use the modified polynomial chaos expansion to simplify the calculation of the statistics of the RHS vector. This is implemented by first expanding $\Delta Z(H)$ in terms of Hermite polynomials using Theorem 1 $\Delta Z(H)=\sum_{i=1}^{K} Z_{i} \Psi_{i}(\eta)$ and then substituting this expansion in (6):

$$
\begin{gathered}
I_{m}(\eta)=Z_{m 0}^{-1} \Delta \Phi_{m}-Z_{m 0}^{-1}\left(\sum_{i=1}^{K} Z_{i} \Psi_{i}(\eta)\right) Z_{m 0}^{-1} \Delta \Phi_{m} \\
+\quad Z_{m 0}^{-1}\left(\sum_{i=1}^{K} Z_{i} \Psi_{i}(\eta)\right) Z_{m 0}^{-1}\left(\sum_{i=1}^{K} Z_{i} \Psi_{i}(\eta)\right) Z_{m 0}^{-1} \Delta \Phi_{m}+.
\end{gathered}
$$

From (13) we observe that the complexity is transformed from solving a large system into doing a large number of matrix multiplications. This characteristic is very appreciable in such a formulation also due to the inherent parallelizability of such an algorithm. Before proceeding with the time and memory complexity analysis it is worth mentioning that in general we are not interested in the entire current vector $I_{m}(\eta)$, but rather just in the current at the ports. For a single port excitation (13) is simplified to:

$$
i_{o}(\eta)=i_{m 0}-\sum_{i} I_{m 0}^{T} Q_{i} \Psi_{i}(\eta)+\sum_{i} \sum_{j} Q_{i}^{T} Z_{m 0}^{-1} Q_{j} \Psi_{i}(\eta) \Psi_{j}(\eta)
$$

where $I_{m 0}=Z_{m 0}^{-1} \Delta \Phi_{m}, i_{m 0}=\Delta \Phi_{m}^{T} I_{m 0}$ and $Q_{i}=Z_{i} I_{m 0}$. Note that we have used the symmetry of the projection coefficient matrices $Z_{i}=Z_{i}^{T}$ in deriving the last expression.

\subsection{Complexity analysis}

\subsubsection{Memory}

Theoretically, we would need to store the LU factors of matrix $Z_{m 0}$ in addition to all the matrices $Z_{i}$. This means that the total memory requirement should be $O\left(N^{2}\right)+O\left(K N^{2}\right)$. However, a careful investigation of (14) reveals that the only way the matrices $Z_{i}$ appear in such equation is implicitly as a matrix vector product $Q_{i}$. Furthermore, the $K$ vectors $Q_{i}$ can be stored offline and be individually recalled during the system assembly without affecting the performance. Hence, only the $O\left(N^{2}\right)$ part is required to reside in memory at a certain point of time. In other words, our algorithm has the same memory complexity $O\left(N^{2}\right)$ as Monte Carlo based approaches and is very memory efficient if compared to the $O\left(N^{4}\right)$ complexity of the standard Neumann expansion, or the $O\left(K^{2} N^{2}\right)$ complexity of the standard stochastic finite element simulation.

\subsubsection{Time}

We will base our analysis on the fact that the mesh impedance matrix is symmetric and the fact that we employ a direct matrix solver since this will enable us to recycle the LU factorization. We first factor the nominal system for a complexity of $O\left(N^{3}\right)$. We then compute $K$ unique matrix-vector products $O\left(K N^{2}\right), K$ vectorvector products $O(K N), K$ system solves (recycling the LU factorization) $O\left(K N^{2}\right)$ and $K^{2}$ vector-vector products $O\left(N K^{2}\right)$. The final complexity is therefore $O\left(N^{3}\right)+O\left(N^{2} K\right)+O\left(N K^{2}\right)=O\left(N^{3}\right)+$ $O\left(N K^{2}\right)$. This means that our algorithm is very time efficient if compared to the $O\left(N^{4}\right)$ complexity of the standard Neumann expansion, the $O\left(N^{3} K^{3}\right)$ complexity of the standard stochastic finite element simulation, or the $O\left(N_{M C} N^{3}\right)$ complexity of the MonteCarlo like algorithms, where $N_{M C}$ is the number of independent simulations.

\subsubsection{Parallelizability}

One of the main advantages of the proposed algorithm is its inherent parallelizability as is evident from (14). Since there is practically no communication required between the different processors, the computational time required to do the different matrix-vector and vector-vector products can be reduced by $N_{P}$, the number of 


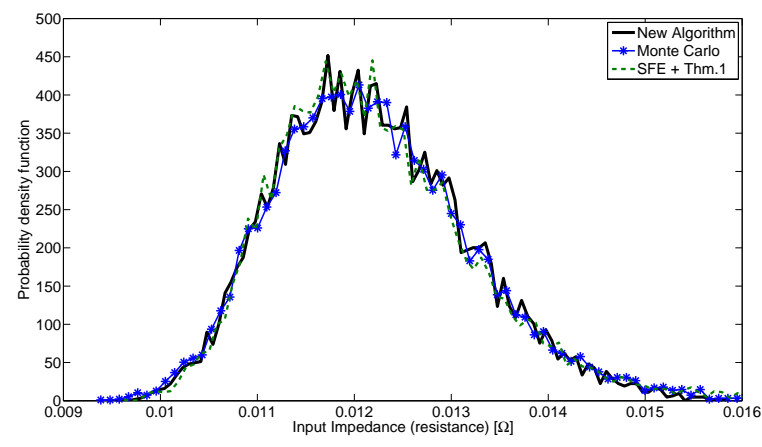

Figure 1: Comparison between the probability density function of the microstrip line obtained from our new algorithm and the reference Monte Carlo simulation.

available processors. Consequently, the final time complexity is $O\left(N^{3}\right)+O\left(N \frac{K^{2}}{N_{P}}\right)$.

\section{RESULTS}

\subsection{Accuracy Validation}

In the first example we verify the accuracy of the proposed method using a single $50 \mu \mathrm{m}$ wide, $0.5 \mathrm{~mm}$ long and $15 \mu \mathrm{m}$ thick microstrip line. The upper surface of the line is highly irregular and is described with a Gaussian random process of standard deviation $\sigma=$ $3 \mu \mathrm{m}$ and correlation length $L_{c}=50 \mu \mathrm{m}$. We use a total of $200 \mathrm{un}$ knowns to model the current density inside of the microstrip line, and a total of 19 independent random variables to model the rough surface. The number of orthogonal polynomials for a second order $(P=2), 19$-variables $(M=19)$ Hermite expansion of the system matrix is computed from (10), and is equal to $K=210$. This structure is simple enough to be accurately discretized and simulated using 10,000 Monte Carlo simulations, and to be simulated using the stochastic finite element method (SFE), provided we use Theorem 1 to compute the coefficients of the expansion. A comparison of the complete statistical distributions of the DC input resistance of the microstrip line as obtained from our full new algorithm, Monte Carlo analysis, and the SFE method with Theorem 1 is demonstrated in Figure 1. Specifically, by full new algorithm we mean using Theorem 1 to obtain the coefficients of the Hermite expansion and the combined Neumann-Hermite algorithm in section 4 to solve the linear system. We observe very good agreement between all techniques. The mean of all three distributions is identically equal to 0.0122 . The standard deviation for both Monte Carlo and SFE is identically equal to 0.001 , while our technique computes the standard deviation 0.00097 , which corresponds to a $3 \%$ error.

We further simulate a similar but longer $(1 \mathrm{~mm})$ microstrip line, with roughness described by correlation length $L_{c}=25 \mu \mathrm{m}$. The total number of random variables required to model such a rough surface is 96 . We fail to simulate such a structure using the SFE, since the required matrix size is $1.9 \times 10^{6}$, which cannot be efficiently stored using current state of the art. However, using our technique we can efficiently compute the input resistance of such an interconnect structure. The average input resistance obtained from our algorithm is $0.0243 \Omega$ as compared to $0.0241 \Omega$ obtained from 10000 Monte Carlo simulations. The standard deviation is $8.64 \times 10^{-4}$ as compared to $8.73 \times 10^{-4}$, demonstrating less than $1 \%$ error. Such results clearly indicate the accuracy of our proposed technique. The computational requirement of both examples are summarized in the first two rows of Table 1.
Table 1: Time and Memory Performance Comparison of Monte Carlo, Stochastic Finite Element (SFE) and the New Algorithm

\begin{tabular}{|c|c|c|c|}
\hline Example & $\begin{array}{c}\text { Technique \& Properties for } \\
\text { 5\% accuracy }\end{array}$ & Memory & Time \\
\hline Short & Monte Carlo, 10,000 & $0.32 \mathrm{MB}$ & 24 min. \\
Microstrip & SFE, $M=19$ & $58 \mathrm{MB}$ & $(12$ days $)$ \\
Line & SFE+Theorem $1, \mathrm{M}=19$ & $58 \mathrm{MB}$ & $120 \mathrm{~min}$. \\
DC only & New Algorithm, M=19 & $0.32 \mathrm{MB}$ & 1.8 min. \\
\hline Long & Monte Carlo, 10,000 & $1.2 \mathrm{MB}$ & 2.4 hours \\
Microstrip & SFE, $\mathrm{M}=96$ & $(72 \mathrm{~GB})$ & - \\
Line & New Algorithm, M=96 & $1.2 \mathrm{MB}$ & 0.5 hours \\
\hline Transm. & Monte Carlo, 10,000 & $10 \mathrm{MB}$ & 16 hours \\
Line & SFE, $\mathrm{M}=105$ & $(300 \mathrm{~TB})$ & - \\
10 freq. & New Algorithm, M=105 & $10 \mathrm{MB}$ & 7 hours \\
\hline Two-trun & Monte Carlo, 10,000 & $121 \mathrm{MB}$ & $(150 \text { hours })^{+}$ \\
Inductor & SFE, $\mathrm{M}=400$ & $(800 \mathrm{~PB})$ & - \\
10 freq. & New Algorithm, $M=400$ & $121 \mathrm{MB}$ & 8 hours \\
\hline
\end{tabular}

\subsection{Computational Complexity Comparison}

In this subsection we compare the computational performance of Monte Carlo based algorithms, SFE simulation and our new algorithm on four different interconnect structures, namely, a microstrip line (200 unknowns, $L_{c}=50 \mu \mathrm{m}$ ), a microstrip line (400 unknowns, $\left.L_{c}=25 \mu \mathrm{m}\right)$, a two-wire transmission line (800 unknowns, $L_{c}=$ $50 \mu \mathrm{m})$ and a 2 turn square inductor (2750 unknowns, $\left.L_{c}=50 \mu \mathrm{m}\right)$.

The comparison results (memory and time requirements) are summarized in Table 1. We do not include the Neumann expansion in our comparison since it does not generate complete distributions and is computationally much more inferior even for computing only the average. All the simulations have been run in MATLAB on Intel Xeon, CPU 3.4GHz, 4-processor, 4GB RAM. Parameter $\mathrm{M}$ in Table 1 indicates the number of independent random variables used to expand the random process, and it corresponds to the dimension of the integrals required for calculating the coefficients of the Hermite expansion when not employing our new Theorem 1. However, using Theorem 1 the dimension of the integral is 4 independent of the number of random variables $M$. The number beside the Monte Carlo simulations indicates the total number of simulation runs. The notion 10 freq. indicates results of a 10 point frequency sweep. The frequency band of interest is from $10^{6}$ to $10^{9} \mathrm{~Hz}$. Any number in brackets indicates estimated values. The ${ }^{+}$ superscript indicates running the simulation on the 4 cores in parallel. It can be immediately inferred from the table that the new proposed algorithm is the only possible alternative if the problem size is large.

\subsection{Effect of Surface Roughness on High Fre- quency Input Impedance}

In this example we show how the proposed technique can be used to simulate surface roughness effects on the current distribution inside of a two turn inductor. The inductor has a side length of $1000 \mu \mathrm{m}$ and cross sectional dimensions of $60 \mu \mathrm{m} \times 15 \mu \mathrm{m}$. The frequency band of interest is from $1 \mathrm{MHz}$ to $1 \mathrm{GHz}$. The inductor is discretized using 2,750 non-uniform segments such that the skin depth at the highest frequency $\sqrt{2 / \omega \mu \sigma}=2 \mu \mathrm{m}$ is accurately captured. We will compare the results obtained from different Gaussian rough surface realizations, i.e. different standard deviations $\sigma$ and different correlation lengths $L_{c}:\left(\sigma=3 \mu \mathrm{m}, L_{c}=50 \mu \mathrm{m}\right),(\sigma=$ $\left.3 \mu m, L_{c}=5 \mu m\right)$ and against a completely smooth and deterministic surface. We have observed that the imaginary part of the input impedance divided by $j \omega$ (inductance) typically decreases by less 


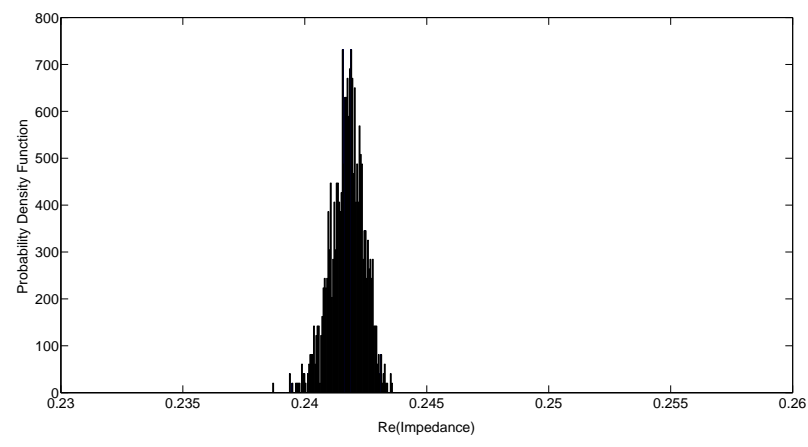

Figure 2: Probability density function of the real part of the input impedance at $1 \mathrm{GHz}$ for correlation length $L_{c}=5 \mu \mathrm{m}$. The resistance of the non-rough surface structure is $0.22 \Omega$, which is 9.8\% smaller than the mean of the obtained distribution.

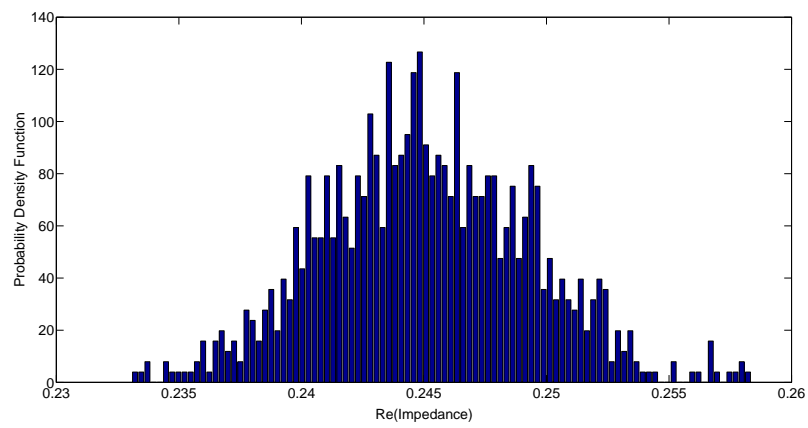

Figure 3: Probability density function of the real part of the input impedance at $1 \mathrm{GHz}$ for correlation length $L_{c}=50 \mu \mathrm{m}$. The resistance of the non-rough surface is $\mathbf{1 1 . 3} \%$ smaller than the mean of the obtained distribution.

than $5 \%$ as a consequence of the surface roughness. On the other hand, the real part of the input impedance (resistance) increases by about $10 \%$ to $20 \%$ as a consequence of the roughness. In addition, we have observed that the standard deviation of the impedance distribution is proportional to the correlation length.

In Figure 2 and Figure 3 the complete probability density functions of the real part of the input impedance at $1 \mathrm{GHz}$ are shown for both small $L_{c}=5 \mu \mathrm{m}$ and large $L_{c}=50 \mu \mathrm{m}$ correlation length, respectively. We observe that the standard deviation of the real part of the input impedance is increased by a factor of 5 when the correlation length increases from $L_{c}=5 \mu \mathrm{m}$ to $L_{c}=50 \mu \mathrm{m}$ for the same standard deviation. The fact that the standard deviation decreases with the decrease of correlation length (increase of surface randomness) is a consequence of the cancellation effects resulting from the distributed nature of the surface.

\section{CONCLUSION}

In this paper we have presented a new methodology to solve large stochastic linear systems. We have developed a new theorem to compute the coefficients of the multivariate Hermite expansion using only low dimensional integrals, resulting in a time complexity that is independent of the number of variables and only dependent on the order of the expansion. Practically speaking, for a typical large multivariate expansion the new theorem provides an improvement in the computation time by 86 orders of magnitude as compared to the standard tensor product rule or by 10 orders of magnitude as compared to the state of the art (Monte Carlo integration or sparse grid integration). Such a theorem is not only useful for our methodology but it can also be applied to any algorithm that relies on expanding a random process, such as the stochastic finite element method. We have also provided a new stochastic simulation technique by merging both the Neumann expansion and the polynomial chaos expansion. The main advantages of the resulting technique are the compact size of the system at any time (unlike SFE) and the ease of calculating the statistics of the high order terms (unlike Neumann expansion). In addition, the new simulation algorithm is parallelizable and can therefore take advantage of the state of the art in processor design. We have demonstrated the computational efficiency of the new methodology by solving problems that were completely untractable before. We have demonstrated that our algorithm can be used to compute the complete probability density function of the input impedance of very large problems (up to 400 random variables) in less then 8 hours using Matlab on a standard 4-core machine and using only 121MB RAM.

\section{Acknowledgment}

The authors acknowledge the support of Cadence Design Systems and of the Interconnect Focus Center, one of five research centers funded under the Focus Center Research Program, a DARPA and Semiconductor Research Corporation program.

\section{REFERENCES}

[1] Z. Zhu, A. Demir and J. White, "A stochastic Integral Equation Method for Modeling the Rough Surface Effect on Interconnect Capacitance" International Conference on Computer Aided Design 2004.

[2] H. Zhu, X. Zeng, W. Cai, J. Xue and D. Zhou, "A sparse grid based spectral stochastic collocation method for variations-aware capacitance extraction of interconnect under nanometer process technology", Design Automation and Test in Europe, 2007.

[3] T. Moselhy and L. Daniel, "Stochastic High Order Basis Functions for Volume Integral Equation with Surface Roughness," EPEP 2007.

[4] Z. Zhu and J. White, "FastSies: A Fast Stochastic Integral Equation Solver for Modeling the Rough Surface Effect" International Conference on Computer Aided Design 2005.

[5] D. Xiu and J. Hesthaven, "High-Order Collocation Method for Differential Equations with Random Inputs" SIAM J. Sci. Comput., 2005.

[6] R. Ghanem and P. Spanos, Stochastic Finite Elements: A Spectral Approach, Spring-Verlag, 1991.

[7] M. Kamon, N. Marques and J. White "Generating compact guaranteed passive reduced-order models for 3-D RLC interconnects" IEEE Trans. on Advanced Packaging, Vol.27, Nov. 2004.

[8] M.Loeve, Probability Theory, Spring-Verlag, 1977.

[9] D. Xiu and G. Karniadakis, "The Wiener-Askey Polynomial Chaos Expansion For Stochastic Differential Equations", SIAM Journal of Scientific Computing, October 2002.

[10] T. Gerstner and M. Griebel, "Numerical Integration using Sparse Grids" Numerical Algorithms, 1998.

[11] D.W. Bolton, "The Multinomial Theorem" The Mathematical Gazette, December 1968. 